\title{
Anterior Decompression and Shortening Reconstruction with a Titanium Mesh Cage through a Posterior Approach Alone for the Treatment of Lumbar Burst Fractures
}

\author{
Tetsuya Suzuki ${ }^{1}$, Eiji Abe ${ }^{1}$, Naohisa Miyakoshi ${ }^{2}$, Hajime Murai ${ }^{1}$, Takashi Kobayashi $^{1}$, \\ Toshiki Abe ${ }^{1}$, Kazuma Kikuchi ${ }^{1}$, Yoichi Shimada ${ }^{2}$ \\ ${ }^{1}$ Department of Orthopedic Surgery, Akita Kumiai General Hospital, Akita, Japan \\ ${ }^{2}$ Department of Orthopedic Surgery, Akita University Graduate School of Medicine, Akita, Japan
}

\begin{abstract}
Study Design: A retrospective study.
Purpose: To examine the efficacy and safety for a posterior-approach circumferential decompression and shortening reconstruction with a titanium mesh cage for lumbar burst fractures.

Overview of Literature: Surgical decompression and reconstruction for severely unstable lumbar burst fractures requires an anterior or combined anteroposterior approach. Furthermore, anterior instrumentation for the lower lumbar is restricted through the presence of major vessels.

Methods: Three patients with an L1 burst fracture, one with an L3 and three with an L4 (5 men, 2 women; mean age,65.0 years) who underwent circumferential decompression and shortening reconstruction with a titanium mesh cage through a posterior approach alone and a 4-year follow-up were evaluated regarding the clinical and radiological course.

Results: Mean operative time was 277 minutes. Mean blood loss was $471 \mathrm{ml}$. In 6 patients, the Frankel score improved more than one grade after surgery, and the remaining patient was at Frankel E both before and after surgery. Mean preoperative visual analogue scale was 7.0 , improving to 0.7 postoperatively. Local kyphosis improved from $15.7^{\circ}$ before surgery to $-11.0^{\circ}$ after surgery. In 3 cases regarding the mid to lower lumbar patients, local kyphosis increased more than $10^{\circ}$ by 3 months following surgery, due to subsidence of the cages. One patient developed severe tilting and subsidence of the cage, requiring additional surgery.

Conclusions: The results concerning this small series suggest the feasibility,efficacy, and safety of this treatment for unstable lumbar burst fractures. This technique from a posterior approach alone offers several advantages over traditional anterior or combined anteroposterior approaches.
\end{abstract}

Key Words: Lumbar spine, Burst fracture, Posterior approach

\section{Introduction}

Thoracolumbar and lumbar burst fractures are common spinal injuries that involve severe instability and intra-canal bony fragments, leading to increased risks of neurological complications and kyphotic deformities. Patients with neu- rological deficits frequently require surgical treatment with spinal instrumentation to relieve pain, address palsy, and stabilize the spine to prevent kyphotic deformity. However, there is controversy about the optimal surgical method of treating burst fractures $[1,2]$.

Some reports have described posterior reconstruction us-

Received Aug 11, 2011; Revised Jan 16, 2012; Accepted Jan 26, 2012

Corresponding author: Tetsuya Suzuki, MD, PhD

Department of Orthopedic Surgery, Akita Kumiai General Hospital,

Nishibukuro, Iijima, Akita 011-0948, Japan

Tel: +81-18-880-3000, Fax: +81-18-880-3040, E-mail: suzuki-te@archosp-1998.com

Copyright (C) 2012 by Korean Society of Spine Surgery

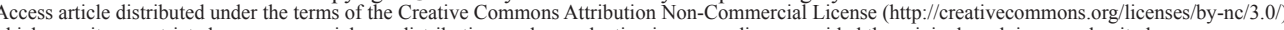
which permits unrestricted non-commercial use, distribution, and reproduction in any medium, provided the original work is properly cited. Asian Spine Journal • pISSN 1976-1902 eISSN 1976-7846 
ing pedicle screws, aiming to reposition canal-compromising fragments by ligamentaxis or remodeling of the canal [3-6]. Posterior surgeries of this type for thoracolumbar burst fractures have reported satisfactory results in fractures involving mild instability [1,2]. Conversely, McCormack et al. [7] proposed thet highly unstable spine fracture with load-sharing scores (LSS) $\geq 7$ should be treated by anterior column reconstruction. For highly unstable thoracolumbar burst fractures, anterior decompression and reconstruction has been developed with a variety of anterior implant systems, which have achieved satisfactory results [8-12].

In cases of highly unstable burst fractures below L4, a combined anterior-posterior approach to decompress the spinal canal and reconstruct the segments is generally necessary, because the presence of common iliac arteries and veins, iliopsoas muscles, and iliac crests restrict the use of anterior instruments on L5 or S1 vertebrae. Most cases of fractures above L3 can, however, be treated using only an anterior or posterior approach $[8,13]$. Anterior-posterior combined surgeries risk increasing operative morbidity due to vascular and pulmonary complications $[14,15]$, particularly in compromised elderly patients $[16,17]$.

One option for the treatment of unstable thoracolumbar and lumbar burst fracture is single-stage posterior corpectomy and reconstruction, using various recently-introduced cages [18-20].

This study reports a novel posterior surgery and outcomes in seven patients with lumbar burst fracture, with and without neurological deficits. In these cases, the damaged vertebrae were sub-totally removed via a posterior approach alone, followed by vertebral replacement with a short mesh cage and pedicle screwing. Details of the technique using sub-total corpectomy and shortening reconstruction with a cage are described.

\section{Materials and Methods}

\section{Methods}

Subjects comprised seven consecutive patients (five men, two women; mean age, 65.0 years; range, 42 to 78 years) with burst fractures of the first, third and forth lumbar vertebrae, which were operatively treated using a posterior approach for sub-total vertebrectomy, followed by replacement with a mesh cage and pedicle screwing between April 2005 and June 2006. Surgical indication for this study was burst fracture in the lumbar spine with difficulty in walk- ing due to paralysis of the lower extremities or severe low back pain, which decreased the patients' quality of daily life. Neurological status was classified using Frankel grades, and functional status was evaluated using a visual analogue scale (VAS) ranging from 0 to 10 for pain of the lower back or legs. All patients were examined radiologically. The extent of instability was graded using LSS, as described by McCormack et al. [7]. Bone mineral density (BMD) in the region of the femoral neck was measured by dual energy $\mathrm{X}$-ray absorptiometry. The angle of kyphosis from the superior endplate of the vertebral body above the fractured level to the inferior endplate of the vertebral body below the affected vertebra was measured on lateral radiograms in the neutral position, before and immediately after surgery, and the final follow-up examination. Instrumentation failure, such as screw-loosening, migration of the cage, and loss of correction were evaluated from anteroposterior and lateral radiograms. Screw-loosening was defined as the existence of $2 \mathrm{~mm}$-widths of clear-zone around any screw, migration of the cage was defined when the upper or lower surface of the cage migrated into the vertebral endplate by more than $2 \mathrm{~mm}$, and loss of correction was recognized when the kyphotic angle was increased by more than $10^{\circ}$ between the initial and the final X-rays. The mean duration of follow-up was 58 months (range, 51 to 65 months).

\section{Surgical technique}

The patient is placed prone on the Hall frame to avoid compression of the vena cava and to decrease thoracolumbar kyphosis. A posterior midline incision is made, extending one to two vertebrae above and below the affected vertebra. The paraspinal muscles are dissected subperiosteally from the spinous process and laminae. The lateral area around the facet joints above and below the fractured level is dissected carefully. The lower half of the lamina above the collapsed vertebra is resected using an osteotome followed by excision of the lamina and bilateral superior articular processes of the affected vertebra using a rongeur. A high-speed diamond burr is used to resect bilateral pedicles, then the fractured vertebral body is drilled laterally and anteriorly. The upper and lower discs are removed, including the cartilaginous endplates. The freed burst fragment with posterior longitudinal ligament are gently detached from the ventral surface of the dura mater, then the area around the conus medullaris or cauda equina is decompressed completely (Fig. 1A). 


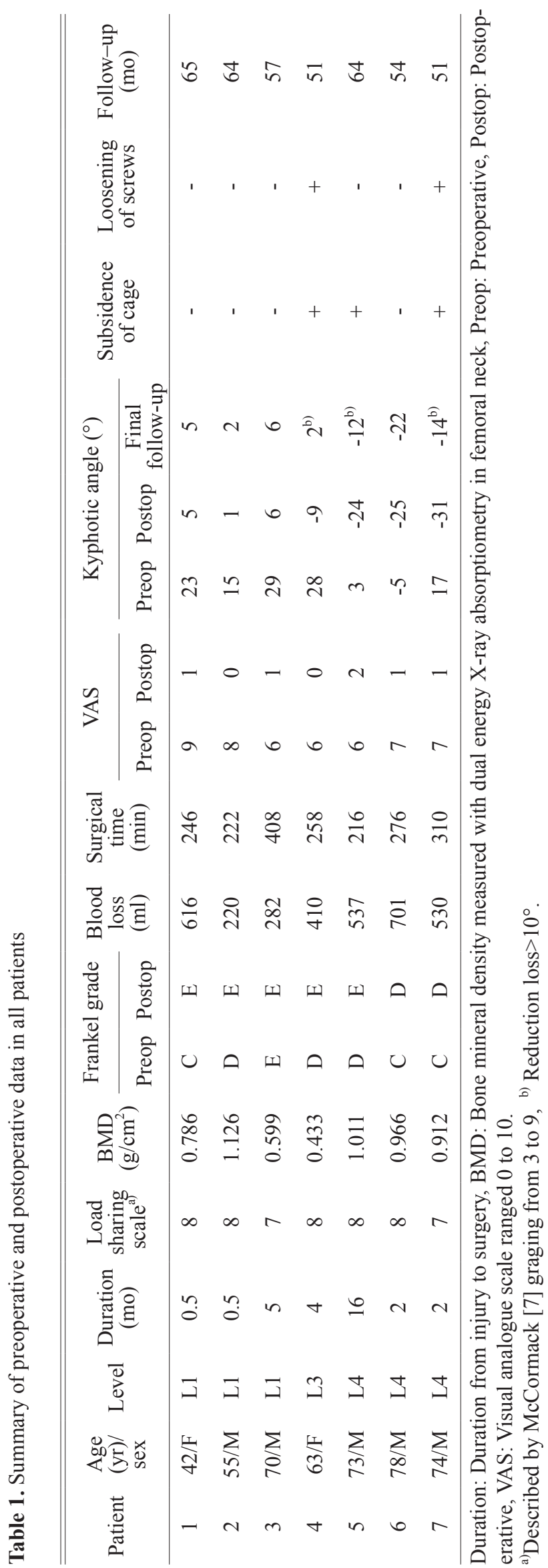

Pedicle screws from the Total Spine System (Mizuho, Tokyo, Japan) are inserted into the one or two vertebrae above and below the lesion, considering the extent of osteoporosis, under lateral fluoroscopic imaging. We used a cylindrical titanium mesh cage (Harms cage with cross-section $22 \times$ $18 \mathrm{~mm}$, height 22 to $30 \mathrm{~mm}$, DePuy, Raynhem, MA, USA) filled with milled local bone. The mesh cage is inserted by gently retracting the nerve roots and dura mater (Fig. 1B). In cases of L4 fracture, the lower surface of the cage is cut obliquely to restore the lordotic alignment. The cage is then aligned longitudinally in the cavity. Once the cage is placed in the center of the endplates, rods are applied to the pedicle screws and the cage is fixed with compressive force to correct angular deformity and shortening of the spinal column, but not restoring the original height of the vertebral body and discs. Augmentation of hooks and cross-links between rods is added if necessary, considering poor bone quality or the level of the injured vertebra. Finally, the remaining milled local bone is embedded around the cage and on the posterolateral side of the column for facet fusion.

\section{Statistical analysis}

Differences in clinical findings (VAS, kyphotic angle) between pre- and postoperatively and at final follow-up were analyzed using paired $t$-tests and repeated analysis of variance. The level of significance was set at 95\%.

\section{Results}

Table 1 lists the demographic data of pre- and postoperative characteristics for each of the seven patients. Mean duration from injury to surgery was 4.3 months (range, 0.5 to 16 months). All patients had LSS $\geq 7$, with a mean score of 7.7. BMD at the femoral neck was relatively low, (mean, $0.833 \mathrm{~g} / \mathrm{cm}^{2}$; range, 0.433 to $1.126 \mathrm{~g} / \mathrm{cm}^{2}$ ). Before surgery, only one patient was neurologically intact, and he continued to have no motor or sensory deficits after surgery. Another six individuals were assessed at Frankel grades $\mathrm{C}$ or $\mathrm{D}$ before surgery. All of these patients improved more than one level after surgery. Mean operative time was 277 minutes (range, 216 to 408 minutes). Mean blood loss was $471 \mathrm{ml}$ (range, 220 to $701 \mathrm{ml}$ ), and no blood transfusions were needed. Mean preoperative pain score VAS was 7.0 (range, 6 to 9). Pain in the lower back or legs was relieved in the early postoperative period for all patients, and postoperative mean VAS was kept at 0.9 (range, 0 to 2) until final follow- 
up. A significant difference was present between pre- and postoperative scores $(p<0.05)$. No perioperative complications such as cerebrospinal fluid leakage, new neural irritation caused by hardware or grafted bone, superficial or deep wound infections, deep venous thrombosis or pulmonary embolism were caused. Local kyphosis was improved from $15.7^{\circ}$ before surgery to $-11.0^{\circ}$ after surgery and progressed to $-4.7^{\circ}$ at final follow-up. A significant difference was seen between kyphotic angle preoperatively and at final followup $(p<0.05)$ in all cases. The total kyphotic correction was $20.4^{\circ}$ on average (range, $13^{\circ}$ to $31^{\circ}$ ). Restored segmental alignment worsened by $>10^{\circ}$ in three patients with L3 and
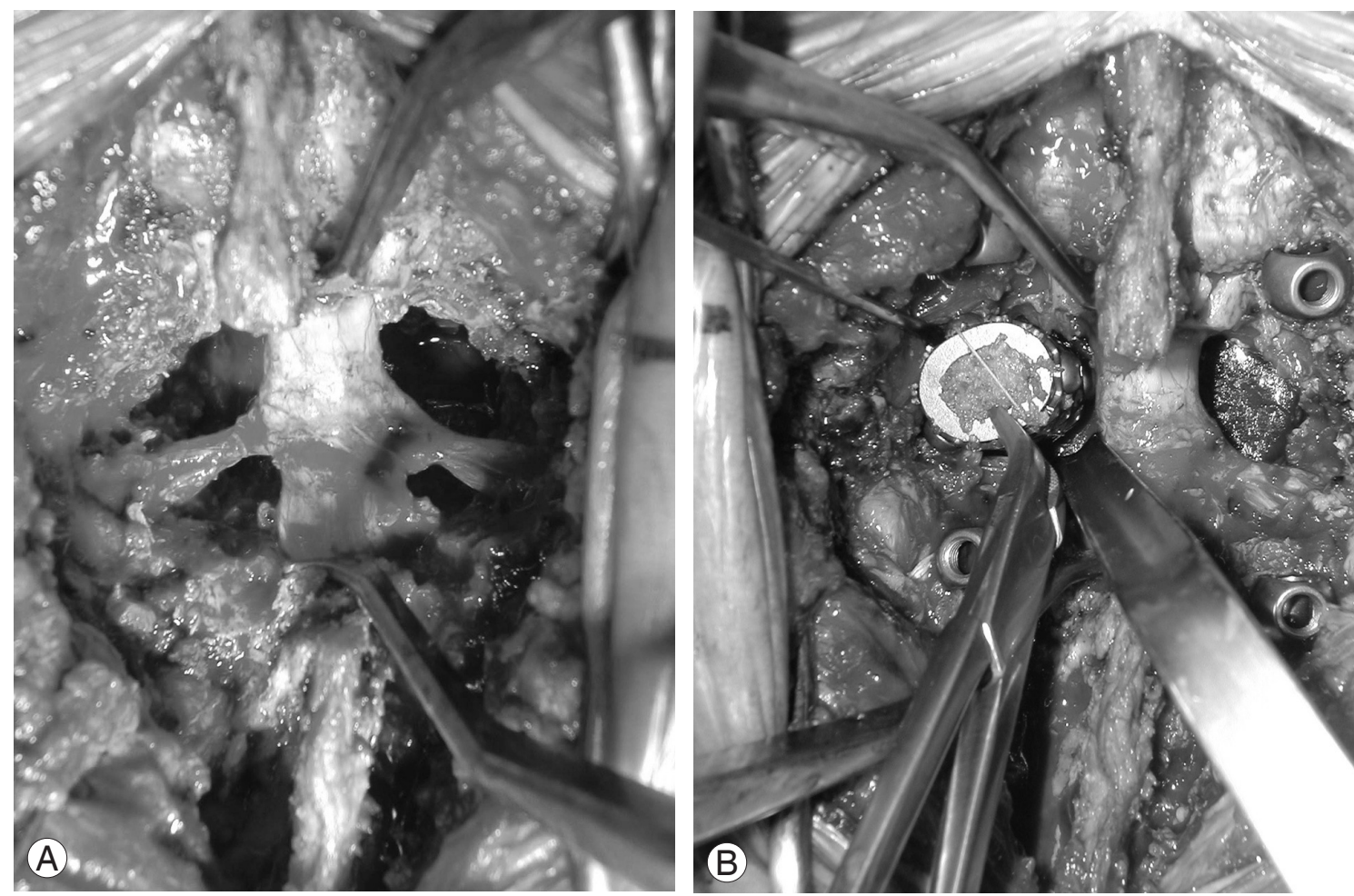

Fig. 1. Photographs of the surgical procedure for L4 burst fracture. (A) Neural elements are decompressed circumferentially after laminectomy and facetectomy followed by transpedicle corpectomy. (B) The mesh cage is inserted axially after first gently retracting the L3 and L4 nerve roots and the dura mater. The mesh cage is placed vertically against the endplates in the cavity.
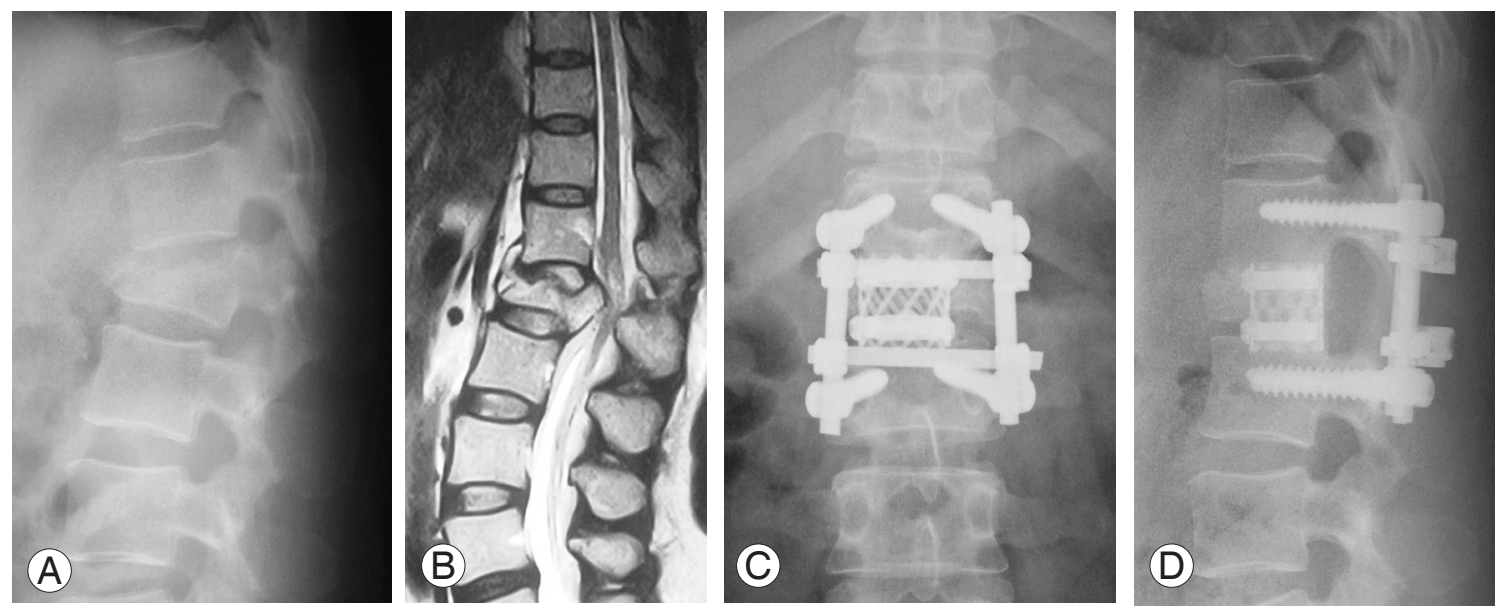

Fig. 2. A 42-year-old woman with L1 burst fracture. (A) Preoperative lateral radiogram showing massive destruction of the vertebra and local kyphosis. (B) Sagittal magnetic resonance imaging showing destruction of upper and lower endplates and compression of the conus medullaris by burst fragment. (C, D) Postoperative anteroposterior and lateral radiogram showing the replaced L1 vertebra with reduced kyphosis. 
L4 fractures within three months after surgery. One patient (case 7) needed additional posterior surgery to extend the pedicle screw fixation and facet fusion, due to severe subsidence and tilting of the titanium mesh cage. Lordotic angle was then maintained until final follow-up.

\section{Case reports}

\section{1) Patient 1}

A 42-year-old woman suffered L1 burst fracture by falling a significant distance in a suicide attempt. The radiogram showed $23^{\circ}$ of local kyphosis with Denis type B fracture (Fig. 2A). Computed tomograms and magnetic resonance imaging revealed massive vertebral destruction and compression of the conus medullaris by bony fragments (Fig.
2B). The patient showed motor-useless palsy and severe pain in both legs (VAS score, 9). Shortening reconstruction with a titanium mesh cage was performed using a posterior approach 12 days after injury (Fig. 2C and 2D). Pain and palsy were relieved in the early postoperative period. The kyphotic angle decreased to $5^{\circ}$ and was maintained until final follow-up. The patient has subsequently returned to her previous occupation with only occasional mild low back pain.

\section{2) Patient 5}

A73-year-old man sustained L4 burst fracture after falling $3 \mathrm{~m}$ from a ladder. He was treated conservatively in another hospital, but the fracture did not heal and deteriorated to an unstable burst fracture with severe low back pain and
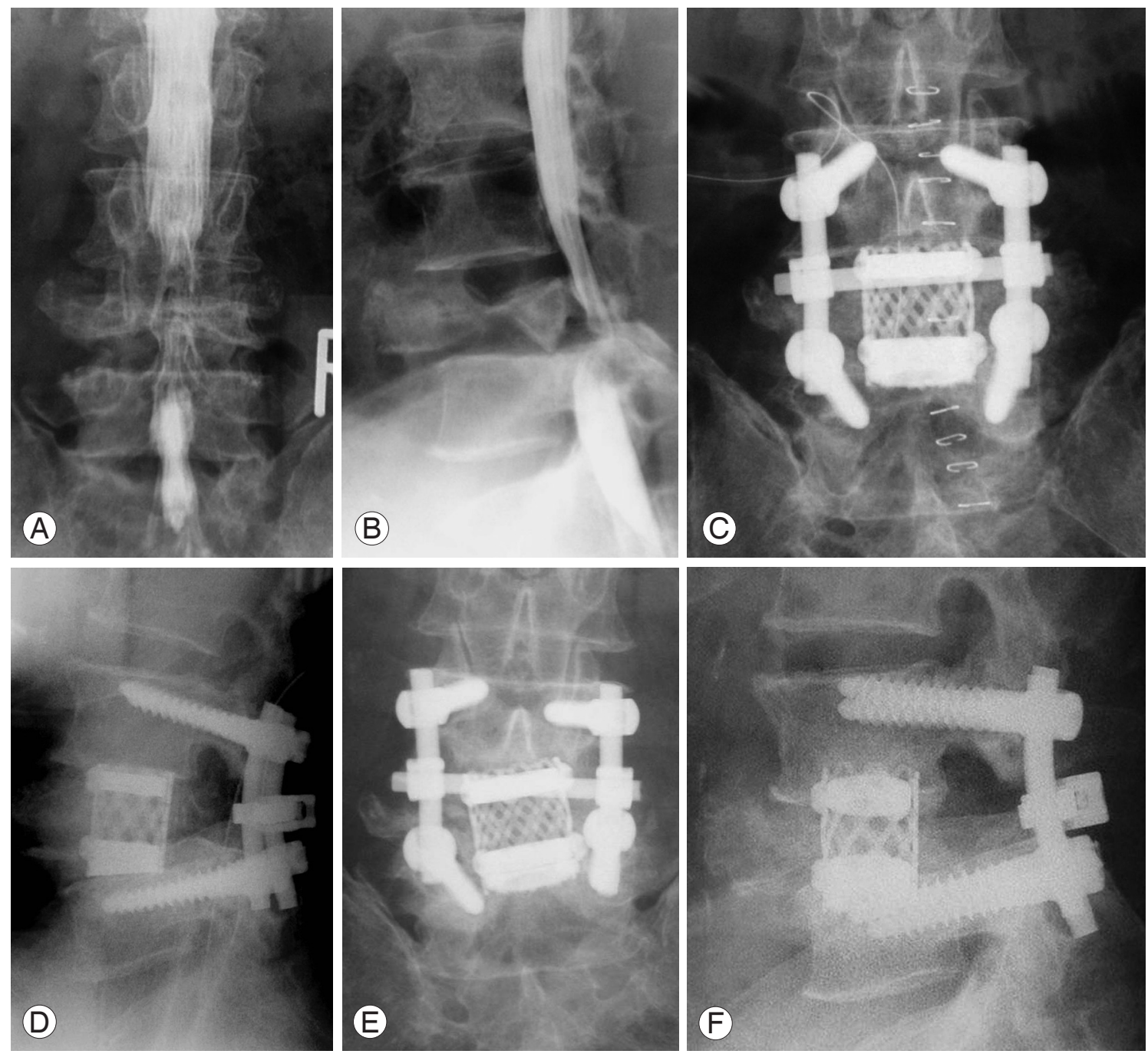

Fig. 3. A 73-year-old man with late collapse of L4 fracture. (A, B) Preoperative anteroposterior and lateral myelogram showing massive collapse of L4 with kyphotic deformity and spinal canal stenosis. (C, D) AP and lateral radiogram immediately after operation, showing lordosis of $24^{\circ}$ between the L3 and L5 vertebrae by reconstruction with the mesh cage and pedicle screws. (E, F) AP and lateral radiogram at 6 months postoperatively, demonstrating subsidence of the cage into the attached endplates causing loss of lordosis. 
numbness in both legs. Radiographic examination of the lumbar spine showed destruction of the L4 vertebra with compression of the dura mater (Fig. 3A and 3B). The patient underwent shortening reconstruction with a cage from a posterior approach using the aforementioned techniques (Fig. 3C and 3D). The numbness in the legs resolved after surgery, but mild low back pain remained for three months.

Follow-up X-ray showed progression of cage subsidence and decreasing lordosis with bone union being achieved after six months (Fig. 3E and 3F).

\section{Discussion}

Many reports have described controversies about treatment for thoracolumbar and lumbar fractures, due to the high frequency of these injuries and the differences in the severity of instability $[1,2,21]$. Three approaches are available for the surgical treatment of thoracic and lumbar burst fracture, using anterior, posterior, or combined methods. No ideal procedure exists, due to the wide variety of clinical and radiological features present in indivisual cases $[1,2,21,22]$.

In cases of severely unstable lumbar burst fractures, anterior column reconstruction and decompression are necessary. To reconstruct the anterior column in lumbar spine, resection of the affected vertebra and strut bone graft or replacement with a cage can be managed from an anterior approach. However, anterior instrumentation for L5 or S1 are restricted by anatomical factors such as the presence of major vessels, the iliopsoas muscles, and iliac crests, so combined posterior instrumentation is definitely needed in cases of L4, 5 fractures $[7,8,13]$.

Recently, single-stage posterior corpectomy and replacement with various types of cage followed by pedicle screw instrumentation for thoracolumbar burst fractures have been reported [18-20]. Sasani and Ozer [20] showed excellent outcomes for corpectomy and replacement with an expandable cage using a posterior approach for the treatment of T8 to L4 burst fracture in non-elderly patients (average age, 40.3 years). They proposed that a single-stage posterior approach may be preferable to the combined anterior-posterior approach and that this procedure is associated with fewer complications than the traditional combined approach. Haiyun et al. [19] reported a similar three-column reconstruction of thoracolumbar fracture above L2 through a single posterior approach with mesh cages and pedicle screws. They noted that use of an adequate shorter nonexpandable cage can provide sufficient biomechanical stability and has benefits in the treatment of thoracolumbar burst fractures. Using an expandable cage allows easier restoration of body height than use of a nonexpandable cage or strut bone. However, shortening reconstruction for unstable burst fracture with a shorter cage appears to offer several advantages: 1) acute spinal column shortening within the safe range increases spinal cord blood flow, which is important for recovery of spinal cord function [23], 2) biomechanical comparisons among different cages have shown no significant differences [24], and 3) resected local bone (affected vertebra, lamina, articular and spinal processes) can maintain bone-grafting inside and around the cage with no complications at the donor site.

We applied this surgical technique for not only the thoracolumbar junction, but also the mid to low lumbar level. The biggest advantage of shortening reconstruction with a titanium mesh cage from a solely posterior approach is that circumferential decompression and fixation containing anterior column reconstruction for mid to low lumbar segments can be introduced as conveniently as for thoracolumbar lesion without anatomical restrictions on instrumentation. Inserting a 22-mm-diameter cage between the upper and lower nerve roots without sacrificing a root may appear technically difficult, but no complications involving cage insertion, such as neural injury or dural tear, were observed in our study. We found that if the surgeon's thumb (with double gloves) can be inserted into the resected vertebral cavity through the inter-nerve space, the cage can be easily introduced without dural or nerve root injuries.

Another difficulty in reconstruction of the mid to low lumbar region is the maintenance of lordosis. The general alignment of the straight thoracolumbar junction spreads the axial load uniformly on the cage, but an unbalanced axial load and shearing force may appear in the lumbar region. In our cases involving mid to low lumbar lesions, patients were elderly at the time of surgery and BMD was relatively low. Although clinical results in those patients with mid to lower lumbar lesions were comparable with those with thoracolumbar lesions, the radiologic results of the former were poorer than the latter because three of four patients in the former group experienced subsidence of the cage and correction loss of more than $10^{\circ}$. These findings suggest that especially in mid to low lumbar burst fractures with low BMD, further modification is essential when using this surgical procedure. Because a single $22 \mathrm{~mm}$ cage may not be enough to work as an anterior support without postopera- 
tive sinking into the adjacent vertebral bodies in the mid or lower lumbar levels, but inserting cages larger than $22 \mathrm{~mm}$ through the nerve roots plexus without injuring the nerve roots seems to be difficult, use of multiple cages may be a solution. Positioning of the cage on the peripheral rim of the vertebral endplate may be another solution. Other types of cages may be required to achieve a wider contact area with the endplates to obtain better stability [25]. Furthermore, multiple sublaminar anchoring with polyethylene tapes may help to stabilize the spinal column.

The posterior surgery techniques in this paper offer considerable advantages, such as a familiar approach for spine surgeons, fewer anatomical limitations due to the presence of major vessels, and circumferential decompression followed by pedicle screwing reconstruction with a reduced risk of complication compared to anterior or combined approaches. This procedure can be applied to all types of lumbar burst fractures, however, the indication should be limited to massive destructed fractures with more than 7-points of LSS. For burst fractures with fewer than 6-points of LSS, less invasive procedures, such as conventional posterolateral fusion, vertebroplasty, or shortening osteotomy with posterior decompression and instrumentation should be selected.

\section{Conclusions}

Although the study population is small, the clinical results of the present study suggest that anterior decompression and shortening reconstruction with a titanium mesh cage using only a posterior approach offers several advantages over traditional anterior or combined anteroposterior approaches. However, further modification are required for this procedure to prevent the subsidence of cages, especially in the mid and lower lumbar lesions.

\section{REFERENCES}

1. Vaccaro AR, Zeiller SC, Hulbert RJ, et al. The thoracolumbar injury severity score: a proposed treatment algorithm. J Spinal Disord Tech 2005;18:209-15.

2. Vaccaro AR, Lim MR, Hurlbert RJ, et al. Surgical decision making for unstable thoracolumbar spine injuries: results of a consensus panel review by the Spine Trauma Study Group. J Spinal Disord Tech 2006;19:1-10.

3. Altay M, Ozkurt B, Aktekin CN, Ozturk AM, Dogan $\mathrm{O}$, Tabak AY. Treatment of unstable thoracolumbar junction burst fractures with short- or long-segment posterior fixation in magerl type a fractures. Eur Spine J 2007; 16:1145-55.

4. Knop C, Fabian HF, Bastian L, Blauth M. Late results of thoracolumbar fractures after posterior instrumentation and transpedicular bone grafting. Spine (Phila $\mathrm{Pa}$ 1976) 2001;26:88-99.

5. Kuner EH, Kuner A, Schlickewei W, Mullaji AB. Ligamentotaxis with an internal spinal fixator for thoracolumbar fractures. J Bone Joint Surg Br 1994;76:107-12.

6. Tezeren G, Kuru I. Posterior fixation of thoracolumbar burst fracture: short-segment pedicle fixation versus long-segment instrumentation. J Spinal Disord Tech 2005;18:485-8.

7. McCormack T, Karaikovic E, Gaines RW. The load sharing classification of spine fractures. Spine (Phila Pa 1976) 1994;19:1741-4.

8. Kaneda K, Taneichi H, Abumi K, Hashimoto T, Satoh $\mathrm{S}$, Fujiya M. Anterior decompression and stabilization with the Kaneda device for thoracolumbar burst fractures associated with neurological deficits. J Bone Joint Surg Am 1997;79:69-83.

9. McDonough PW, Davis R, Tribus C, Zdeblick TA. The management of acute thoracolumbar burst fractures with anterior corpectomy and Z-plate fixation. Spine (Phila Pa 1976) 2004;29:1901-8.

10. Dai LY, Jiang LS, Jiang SD. Anterior-only stabilization using plating with bone structural autograft versus titanium mesh cages for two- or three-column thoracolumbar burst fractures: a prospective randomized study. Spine (Phila Pa 1976) 2009;34:1429-35.

11. Dvorak MF, Kwon BK, Fisher CG, Eiserloh HL 3rd, Boyd M, Wing PC. Effectiveness of titanium mesh cylindrical cages in anterior column reconstruction after thoracic and lumbar vertebral body resection. Spine (Phila Pa 1976) 2003;28:902-8.

12. Sasso RC, Best NM, Reilly TM, McGuire RA Jr. Anterior-only stabilization of three-column thoracolumbar injuries. J Spinal Disord Tech 2005;18 Suppl:S7-14.

13. Suzuki T, Abe E. Spinal instrumentation for thoracolumbar injuries: Causes and prevention of their revision surgery. Spine Spinal Cord 2009;22:851-8.

14. Korovessis P, Baikousis A, Zacharatos S, Petsinis G, Koureas G, Iliopoulos P. Combined anterior plus posterior stabilization versus posterior short-segment instrumentation and fusion for mid-lumbar (L2-L4) burst fractures. Spine (Phila Pa 1976) 2006;31:859-68.

15. Payer M. Unstable burst fractures of the thoraco- 
lumbar junction: treatment by posterior bisegmental correction/fixation and staged anterior corpectomy and titanium cage implantation. Acta Neurochir (Wien) 2006;148:299-306.

16. Miyakoshi N, Shimada Y, Abe E, Suzuki T. Effects and complication measures of the instrumentation surgery for spinal deformity in the elderly. Orthop Surg Traumatol 2010;53:1043-51.

17. Baron EM, Albert TJ. Medical complications of surgical treatment of adult spinal deformity and how to avoid them. Spine (Phila Pa 1976) 2006;31(19 Suppl):S106-18.

18. Ayberk G, Ozveren MF, Altundal N, et al. Three column stabilization through posterior approach alone: transpedicular placement of distractable cage with transpedicular screw fixation. Neurol Med Chir (Tokyo) 2008;48:8-14.

19. Haiyun Y, Rui G, Shucai D, et al. Three-column reconstruction through single posterior approach for the treatment of unstable thoracolumbar fracture. Spine (Phila Pa 1976) 2010;35:E295-302.

20. Sasani M, Ozer AF. Single-stage posterior corpectomy and expandable cage placement for treatment of tho- racic or lumbar burst fractures. Spine (Phila Pa 1976) 2009;34:E33-40.

21. Patel AA, Vaccaro AR, Albert TJ, et al. The adoption of a new classification system: time-dependent variation in interobserver reliability of the thoracolumbar injury severity score classification system. Spine (Phila Pa 1976) 2007;32:E105-10.

22. Verlaan JJ, Diekerhof CH, Buskens E, et al. Surgical treatment of traumatic fractures of the thoracic and lumbar spine: a systematic review of the literature on techniques, complications, and outcome. Spine (Phila Pa 1976) 2004;29:803-14.

23. Kawahara N, Tomita K, Kobayashi T, Abdel-Wanis ME, Murakami H, Akamaru T. Influence of acute shortening on the spinal cord: an experimental study. Spine (Phila Pa 1976) 2005;30:613-20.

24. Pflugmacher R, Schleicher P, Schaefer J, et al. Biomechanical comparison of expandable cages for vertebral body replacement in the thoracolumbar spine. Spine (Phila Pa 1976) 2004;29:1413-9.

25. Lee SW, Lim TH, You JW, An HS. Biomechanical effect of anterior grafting devices on the rotational stability of spinal constructs. J Spinal Disord 2000;13:150-5. 